\title{
Intentional controlled islanding: when to island for power system blackout prevention
}

DOI:

10.1049/iet-gtd.2017.1526

Document Version

Accepted author manuscript

Link to publication record in Manchester Research Explorer

\section{Citation for published version (APA):}

Fernández-Porras, P., Panteli, M., \& Quiros-Tortos, J. (2018). Intentional controlled islanding: when to island for power system blackout prevention. IET Generation, Transmission and Distribution, 12(14), 1-8.

https://doi.org/10.1049/iet-gtd.2017.1526

\section{Published in:}

IET Generation, Transmission and Distribution

\section{Citing this paper}

Please note that where the full-text provided on Manchester Research Explorer is the Author Accepted Manuscript or Proof version this may differ from the final Published version. If citing, it is advised that you check and use the publisher's definitive version.

\section{General rights}

Copyright and moral rights for the publications made accessible in the Research Explorer are retained by the authors and/or other copyright owners and it is a condition of accessing publications that users recognise and abide by the legal requirements associated with these rights.

\section{Takedown policy}

If you believe that this document breaches copyright please refer to the University of Manchester's Takedown Procedures [http://man.ac.uk/04Y6Bo] or contact uml.scholarlycommunications@manchester.ac.uk providing relevant details, so we can investigate your claim.

\section{OPEN ACCESS}




\title{
Intentional Controlled Islanding: When to Island for Power System Blackout Prevention
}

\author{
P. Fernández-Porras, M. Panteli, Member, IEEE, and J. Quirós-Tortós, Member, IEEE
}

\begin{abstract}
Power systems are prone to cascading outages leading to large-area blackouts with significant social and economic consequences. Intentional controlled islanding (i.e., the controlled separation of the system into sustainable islands) has been recently suggested as an effective strategy to mitigate these catastrophic events. To ensure a correct separation, nonetheless, it is crucial to define a suitable time to split the system (i.e., to answer the when to island question). To consider the probability of the event, the reliability of the system components, as well as the reliability of the information and communication technologies and the potential economic costs of the event, answering the above question within a risk-based framework becomes critical. To date, however, this has not been done. This paper proposes a riskbased methodology to define a suitable time to split the system following a severe event. This methodology complements the wellstudied where to island question, thus resulting in an integral solution of the islanding problem. To illustrate the solution, the IEEE 118-bus dynamic system is adopted considering realistic security criteria. Simulation results demonstrate the effectiveness and flexibility of the risk-based methodology in identifying a suitable time for the creation of islands, which, in turn, results in the prevention of blackouts that would otherwise be obtained.
\end{abstract}

Index Terms -Blackout, intentional controlled islanding, power systems, risk assessment, system splitting.

\section{INTRODUCTION}

$\mathbf{I}$ NTERCONNECTED power systems are operated under additional stress to meet the growing demand as well as to accommodate high penetrations of intermittent renewable energy resources [1]. Although this responds to the economic pressure of electricity markets and satisfies environmental targets from governments, it increases the likelihood of cascading outages leading to large-area power system blackouts [2].

Given the social and economic impacts of these catastrophic events (e.g., the 2003 United States-Canada and the 2003 Italian blackouts resulted in economic losses of billions of dollars [3]), transmission systems operators (TSOs) and governments are interested in developing control strategies capable of mitigating the corresponding cascading outages.

Intentional controlled islanding (ICI) has been recently suggested as a corrective, adaptive control action to effectively split the power system into self-sustained islands. There are two main aspects in ICI: $i$ ) where to island, and $i i$ ) when to island. While the former aims to find the optimal set of transmission lines that must be disconnected to divide the

P. Fernández-Porras and J. Quirós-Tortós are with the EPERLab, School of Electrical Engineering, University of Costa Rica, San José 115012060, Costa Rica. M. Panteli is with the School of Electrical and Electronic Engineering, The University of Manchester, Manchester M13 9PL, UK. Emails: jpablofp20@gmail.com, mathaios.panteli@manchester.ac.uk, jairoquirostortos@ieee.org. system into electrically-isolated islands, the latter seeks to define the most appropriate time for islanding. Given that the success of an ICI scheme is highly dependent on the timely implementation of the islanding solution [4]-[6], this work focuses on addressing the question of when to island.

The majority of research efforts have been focused on the where to island question [7]-[9]. Only a few studies have been carried out to try to answer the when to island aspect [4]-[6]. These works use the slow coherency theory [4], decision trees [5] and prony-based methods [6]. Analyzing the rotor angle of machines, they compare the current dynamics with previous information to try to predict future scenarios. While this can be effective in some cases, the existing works cannot cater for unexpected system changes (e.g., sudden line disconnections) and unpredictable events which can result in incorrect system islanding. In addition, their computational burden may inhibit their adoption as real-time tools as the processing of data and comparisons needed can be significantly intensive. Therefore, a method that adopts real-time information to rapidly determine the most suitable time for islanding is required.

Intentional controlled islanding has been recently classified as a System Integrity Protection Scheme (SIPS) [10]. Thus, there is a growing interest from TSOs for assessing their reliability and risk (i.e., the probability of the event multiplied by its impact). Although risk assessments (that consider the probability of the event, the reliability of both the grid components and information and communication technologies, as well as the potential economic costs of the event) have been done for other SIPSs (e.g., generation rejection) [11], this study has not yet been undertaken for ICI schemes.

This paper proposes a risk-based methodology to define the most suitable time to split the system. Following a severe event, the methodology is iteratively executed (i.e., every predefined time sample) to determine the risk of the system separated by an islanding solution [9]. The methodology then compares the risk of the system without and with islanding in a real-time fashion (i.e., within the time frame of milliseconds). When the risk without islanding becomes larger than the risk with islanding, the methodology defines this time as the most suitable one to actually split the grid. The simplicity, scalability and efficiency of the methodology will help operators in the decision-making process of when to island the system to mitigate cascading outages. The methodology complements the well-studied where to island, thus resulting in an integral solution of the islanding problem. It is important to mention that this methodology expands the previous approach [12] by incorporating a real-time evaluation, incorporating a realistic impact criteria for the risk assessment and considering realworld considerations for the security criteria of power systems. 
To illustrate the integral solution, this work uses the dynamic model of the IEEE 118-bus test system considering time-domain simulations and realistic security criteria applied by TSOs. In particular, those from the Costa Rican TSO, which is based on the North American Electric Reliability Corporation (NERC), is adopted. To adequately quantify the risk associated with the ICI scheme, the probability of the event, the reliability of the grid components, the reliability of the information and communication technologies, as well as the potential economic costs of the event are based on the values available in the literature and feedback from the local TSO. Simulation results demonstrate the effectiveness and flexibility of the proposed risk-based methodology in identifying the most suitable time for the creation of islands, which, in turn, results in the prevention of large-area power system blackouts that would otherwise be obtained.

\section{BACKGROUND}

This section initially presents the security criteria and the protection schemes adopted by the TSO in Costa Rica. Key aspects associated with the risk assessment of ICI schemes are then discussed. The spectral clustering-based islanding algorithm [9] adopted here to determine the optimal solution for the "where to island" problem is finally presented.

\section{A. Protection Schemes and Security Criteria in Costa Rica}

Typical protection schemes in Costa Rica adopt a primary protection and a back-up protection. While the former is the first line of defense of the power system (i.e., the closest CBs trigger as soon as the fault is detected), the latter operates only if the primary protection fails (i.e., a delay is considered). The back-up protection can be local (i.e., only the CBs in the same substation trigger) or remote (i.e., CBs in all substations that can feed the fault trigger). The delay associated with the local back-up protection is lower than that of the remote. The operation of a remote back-up protection is considered as an extreme event and it is very likely to lead to a blackout.

The TSO in Costa Rica defines for its security criteria three categories of faults: unique, multiple and severe. Each of them has a different consequence in the system [13]. Unique faults refer to a single-phase to ground or a three-phase fault cleared by a primary protection (i.e., only the affected component, e.g., line, transformers, is disconnected) and no other control actions (e.g., load shedding) are allowed. The TSO, on the other hand, defines a severe fault as an event associated with a single-phase to ground or a three-phase fault that is cleared by a back-up protection (i.e., more than one component of the network is disconnected). Under a severe fault, the TSO might allow the implementation of load shedding schemes, and other control strategies (e.g., controlled islanding) to avoid a largearea blackout in the Costa Rican power system.

\section{B. Risk Assessment}

Assessing the risk of the system has become critical as power systems are operated close to their stability limits. In the context of islanding, this risk assessment is even more important given that this adaptive control action is the last resort to prevent large-area blackouts. In general, the risk, $R$, of an electrical event, $E$, is defined as follows [14]:

$$
R=P(E) \times I
$$

where, $P(E)$ is the probability of occurrence of the event $E$ and $I$ its impact on the network (e.g., amount of lost load).

However, when ICI schemes are adopted, it is important to consider not only the risk of the electrical event, but also the inherent risk of the ICI scheme (as it has been done for other SIPS). This, in turn, results into three risk assessments: (i) risk of a desirable operation (i.e., success); (ii) risk of an undesirable operation (i.e., spurious); and (iii) risk of a failure to operate (i.e., failure). It must be noted that these risk assessments are associated with the possible operational modes of SIPS, i.e., success, failure, and spurious operation.

While a successful operation occurs when the ICI splits the system as design, a spurious activation of the scheme typically happens when it operates incorrectly, i.e., when it is not required. In addition, an ICI scheme failure to operate will not split the power system even though this is required. It is critical to note that a spurious operation and/or the failure to operate of an ICI scheme can be caused by several factors (e.g., hardware and software failures, faulty design logic, and human error). Taking into account these aspects will lead to a better assessment of the corresponding risk of an ICI scheme.

Given the three operational modes of an ICI scheme (i.e., success, spurious and failure), to quantify the corresponding probabilities of adopting this control action it is critical to take into account the interactions between the electrical components (e.g., circuit breakers and protection devices) and Information and Communications Technologies (ICT, e.g., software and communications). For instance, while the control logic of the ICI scheme might operate as designed, the actuators might not actually trigger the action, thus leading to a failure operation of the scheme. To quantify the probabilities associated with the ICI scheme, this work assesses the probability of failure on demand (PFD) and the probability of fail-safe (PFS, i.e., the probability of ICI incorrect operations).

In this paper, the PFD and PFS are determined using fault tree analysis (FTA) given its flexibility, simplicity and, more importantly, as it allows assessing the combination of events that can occur in the network. FTA is a systematic method for identifying the combination of events that can lead to the top event of the fault tree, i.e., ICI undesirable operation [15].

To quantify the impact to be used in each of the aforementioned risk assessments, this study uses the cost associated with the amount of load shedding as a result of the ICI operation. For instance, if the ICI scheme splits the system into islands when not required, then the cost of the corresponding load shedding required to retain the stability of the islands is determined and this value is adopted as the impact in the network when ICI scheme is activated.

\section{Spectral Clustering-Based Islanding}

The aim of this paper is to introduce a risk-based methodology to determine a solution for the when to island problem. 
To demonstrate its effectiveness, however, it is critical to complement the proposed risk-based methodology with a solution that provides an answer for the where to island (when the risk-based methodology defines the most suitable time for islanding, the systems must be split using an islanding solution). Therefore, this section summarizes the ICI methodology proposed in [9] to determine an islanding solution for the where to island problem - the islanding solution found by any other methodology can also be adopted.

In order to create electrically separated and sustainable islands, the work in [9] minimizes the power flow disruption while ensuring that each island contains only coherent generators (generators that oscillated similarly). It also excludes from the search space critical lines that cannot be split.

The ICI methodology is based on spectral clustering (a very effective and efficient graph theoretic approach to split a graph into a number of subgraphs [16]). It uses the eigenvalues and eigenvectors of a (Laplacian) matrix that represents the power flows between two connected branches in the power system [9]. In summary, the following steps are executed to determine the set of transmission lines that splits the electrical network into $k$ electrically-isolated islands [9]:

1) Build a graph $G$ that represents the power flow of the network (with $n$ buses) at the moment of splitting (using the actual power flow at the time of islanding).

2) Compute the eigenvectors $\phi_{1}, \phi_{2}, \cdots, \phi_{k}$ associated with the $k$ smallest eigenvalues of the normalized Laplacian $L_{N}$, which is defined as follows [16]:

$$
\left[L_{N}\right]_{i j}= \begin{cases}1, & \text { if } i=j \\ \frac{-w_{i j}}{\sqrt{d_{i} d_{j}}}, & \text { if } i \neq j \text { and } i j \text { is a branch } \\ 0, & \text { otherwise. }\end{cases}
$$

where $w_{i j}$ is the average power flow between buses $i$ and $j$ and $d_{i}=\sum_{j=1}^{n} w_{i j}$ is the degree of the node [16].

3) Place the eigenvectors $\phi_{1}, \phi_{2}, \cdots, \phi_{k}$ as columns to create $X=\left[\phi_{1}, \phi_{2}, \cdots, \phi_{k}\right] \in \mathbb{R}^{n \times k}$.

4) Normalize each row $x_{i} \in X$ to compute $y_{i}$ which forms the rows of $Y \mathbb{R}^{n \times k}$. The vectors $y_{i}$ represent the buses of the system on the sphere $\mathbb{S}^{k-1}$ (see [9] for details).

5) Define the points $y_{i}$ that represent the generation-buses as the centroids on $\mathbb{S}^{k-1}$.

6) Group every load-bus with the nearest generation-bus based on the proximity between the vectors on $\mathbb{S}^{k-1}$.

7) Group the clusters that contain the coherent generators.

\section{Risk-BASED METhodology}

\section{A. Overview of the Methodology}

Fig. 1 shows the typical time-line implementation of the proposed methodology, which uses the risk-based approach to determine when to island while also considering the where to island aspect of the ICI. An efficient ICI algorithm (e.g., the one in [9]) for determining a where to islanding solution is carried out every pre-defined time sample through an online evaluation that uses the actual system state. The solution is then adopted in the risk-based approach (formally introduced

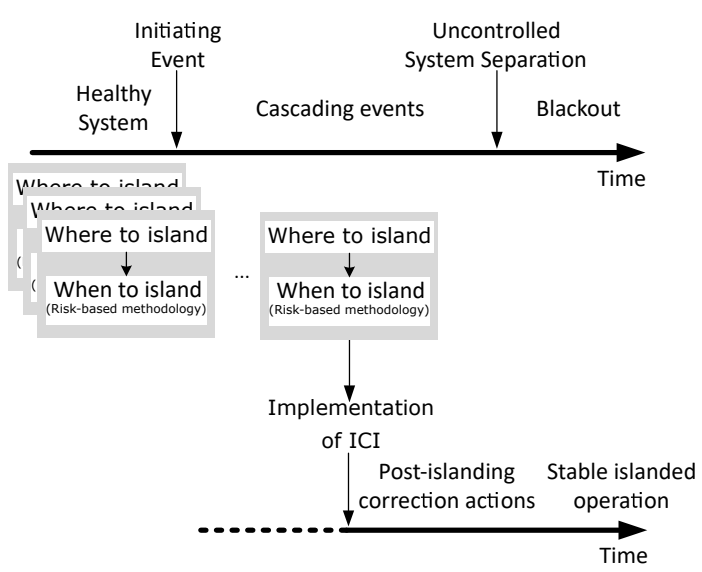

Figure 1. Time-line showing the implementation of the methodology

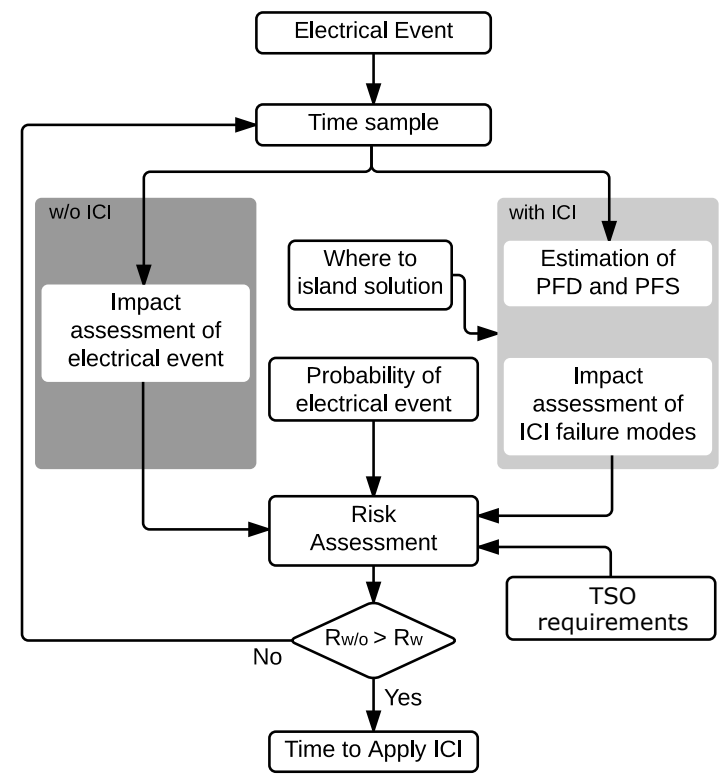

Figure 2. Proposed Methodology

below) to assess whether the system requires to be split or not. If it is determined that the system requires islanding, then the corresponding islanding solution is implemented.

\section{B. Risk-Based Approach}

To define the most suitable moment to split the system, the methodology simultaneously compares the overall risk of the system without and with islanding (see Fig. 2). The risk without islanding (w/o ICI, left side of the flowchart) is quantified considering the impact of the electrical event, as well as the probability of this to happen. In this work, the impact without islanding, $I_{w / o}$, is defined as follows:

$$
I_{w / o}=L_{l} \times V o L L
$$

where $L_{l}$ is the amount of lost load product of the electrical event, and $V o L L$ is the value of lost load, i.e., the economic cost of each MWh that has been lost. It is important to mention that for the application of the proposed methodology the $V o L L$ is considered invariant on time since all assessments are performed in a time frame of seconds and for the variation of this value a long-term time frame is required [11]. 


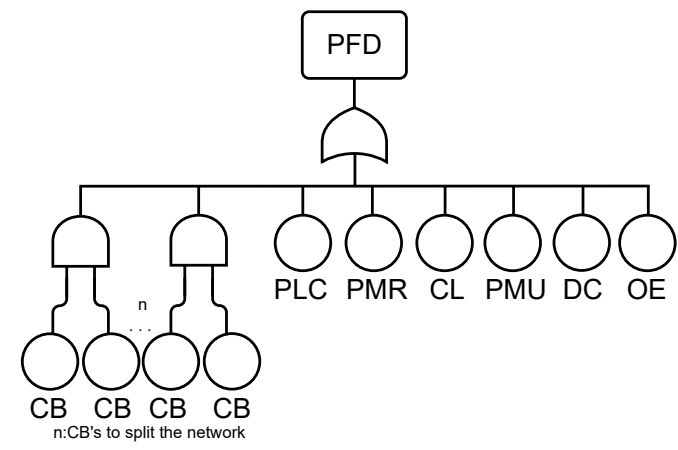

Figure 3. Fault tree for PFD

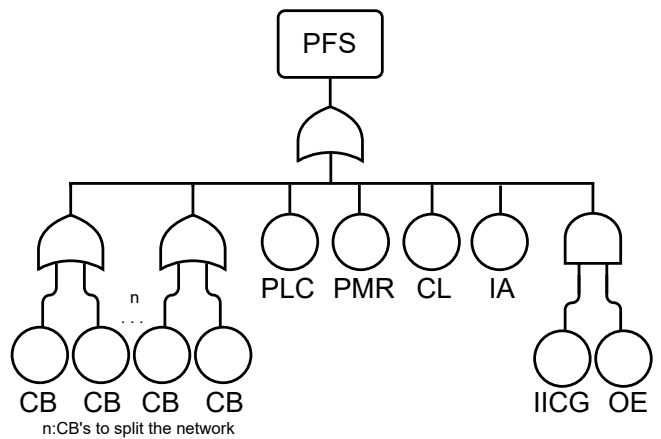

Figure 4. Faul tree for PFS

In parallel to the risk assessment without islanding, the methodology calculates the risk of the system with the ICI scheme in operation (with ICI, right side of the flowchart). To determine this risk, the PFD and PFS, as well as the impact of adopting the islanding solution (i.e., the one obtained with the spectral clustering-based methodology), must be estimated.

To determine the PFD and PFS, this work proposes the fault trees shown in Fig. 3 and Fig. 4. For details of the FTA see section II-B and [15]. To determine these probabilities, Boolean logic is used. It is important to note that these fault trees consider the interactions of different components and factors: circuit breakers (CBs), programmable logic controllers (PLC), power measurement relays (PMR), communication links (CL), incorrect activations (IA, i.e., any other activation that is not associated with the spurious operation of the ICI), phasor measurement units (PMU), DC power supply (DC), operating errors (OE) and incorrect identification of coherent groups (IICG). Other components and factors can also be included, but these are considered as the critical ones that can lead to failure and/or spurious operation of an ICI scheme.

While estimating the above probabilities, the methodology quantifies the impact with islanding, $I_{w}$, resulting from splitting the system according to the ICI solution obtained. This is carried out considering the load shedding for a success and spurious operation, $L_{s u c}$ and $L_{\text {spur }}$, respectively, required to maintain the system within the stability margins after islanding. Given the three operational modes of an ICI scheme, the impact with islanding is estimated as follows:

$$
I_{w}=\left\{\begin{array}{l}
I_{\text {suc }}=L_{\text {suc }} \times V o L L \\
I_{\text {fail }}=L_{l} \times V o L L \\
I_{\text {spur }}=L_{\text {spur }} \times V o L L
\end{array}\right.
$$

where $I_{s u c}$ is the impact of a successful operation, $I_{\text {fail }}$ is the impact of a failure operation, and $I_{\text {spur }}$ is the impact of a spurious operation. It is important to note that a failure operation of the ICI scheme results in the not creation of islands, i.e., the system remains unstable and this is likely to end in a blackout, as in the case w/o ICI. Hence, the impact of this operation mode results from the lost load, $L_{l}$. Despite load shedding has been adopted here as an impact index, any impact can be included in the proposed approach. This represents a key factor with respect to other works, since depending on the TSO requirements the methodology can be easily adapted.

When the above estimations are finished, i.e., when all the impacts and the probabilities have been computed without and with islanding, the risk assessment takes place. The probability of the occurrence of the electrical event, $P(E)$, threatening the network stability is also taken into account in this calculation, which serves as an input variable to the risk assessment.

Therefore, the risk of the power system without and with the ICI scheme activated and in operation, $R_{w / o}$ and $R_{w}$, respectively, are computed as follows [17]:

$$
\begin{gathered}
R_{w / o}=P(E) \times I_{w / o} \\
R_{w}=R_{\text {suc }}+R_{\text {fail }}+R_{\text {spur }} .
\end{gathered}
$$

where,

$$
\begin{gathered}
R_{\text {suc }}=P(E) \times(1-P F D) \times I_{\text {suc }} \\
R_{\text {fail }}=P(E) \times P F D \times I_{\text {fail }} \\
R_{\text {spur }}=(1-P(E)) \times P F S \times I_{\text {spur }}
\end{gathered}
$$

and $R_{\text {suc }}, R_{\text {fail }}$, and $R_{\text {spur }}$, are the risks of a successful, failure, and spurious operation, respectively.

The methodology finally compares the risk without $\left(R_{w / o}\right)$ and with $\left(R_{w}\right)$ the ICI scheme. A $R_{w / o}$ smaller than a $R_{w}$ indicates that the ICI solution (found using the spectral clustering-based methodology) must not be adopted (islanding will have a bigger effect on the system). On the other hand, a $R_{w / o}$ larger than $R_{w}$ highlights that islanding must be undertaken as this leads to a lower risk.

The implementation of islanding is carry out sending signals to open the CBs found by the ICI solution. It must be mentioned that signals to remain close are also sent to the other CBs of the system. The most suitable time to split the system occurs, based on the proposed methodology, when $R_{w / o}$ becomes larger than $R_{w}$ for the first time. This thus gives a solution to define the time to implement an ICI scheme, i.e., an answer for the when to island aspect of the islanding problem.

\section{RESUlts}

This section presents the results of implementing the riskbased methodology on the IEEE 118-bus test system shown in Fig. 5. Time-domain simulations, considering the dynamic parameters of generators provided in [18], are carried out to 
Table I

COMPONENTS RELIABILITY DATA

\begin{tabular}{ccc}
\hline Component & PFD & PFS \\
\hline CB & 0.00091 & 0.0018 \\
PLC & 0.00033 & 0.0067 \\
PMR & 0.00026 & 0.00052 \\
PMU & 0.00026 & 0.00052 \\
OE & 0.000046 & 0.000091 \\
CL & 0.0001 & - \\
DC & 0.00033 & - \\
IA & - & 0.00052 \\
IICG & - & 0.0002 \\
\hline
\end{tabular}

demonstrate the effectiveness of the proposed integral solution. To realistically assess the performance of the system, the simulations consider the security criteria established by the TSO in Costa Rica. The dynamic simulations are performed in DIgSILENT PowerFactory [19], and the methodology is implemented in MATLAB [20]. The time sample used in the simulations is equal to $10 \mathrm{~ms}$, as this is the time required for the whole methodology to run all the calculations and determine the solution.

The robustness of the proposed methodology is illustrated considering two case studies: when islanding is not required, and when islanding is required to avoid a blackout. The effects of a delayed islanding implementation are discussed for the latter scenario. This work adopts the individual PFD and PFS of the grid components and factors, obtained from the literature and discussed with the Costa Rican TSO, shown in Table I.

\section{A. Case Study I: Islanding not Required}

The first case study considers a unique contingency that is cleared by the primary protection, i.e., the fault has no subsequent consequences on the system and only one component is disconnected. It is considered that at time $t=0.1 \mathrm{~s}$, a three phase-to-ground fault occurs near bus 74 at line $74-75$, and it is cleared by opening the faulty line $74-75$ at $t=0.2 \mathrm{~s}$.

Fig. 6(a) clearly shows that the rotor angle of the 19 generators remain coherent and all of them with similar pre and post-fault values. Fig. 6(b) highlights that despite the generators accelerate during the fault, they recover (at around $t=1 s$ ) to the value of $1 p . u$. after the fault is cleared. Fig. 6(c) finally shows that the voltages of the 118 buses of the system remain between $0.95 p . u$. and $1.05 p . u$., limits established by the TSO in Costa Rica. Therefore, and from a dynamic point of view, it can be concluded that the system is stable for this relatively small disturbance and that it does not require to be split into islands.

To demonstrate the effectiveness of the risk-based methodology on determining that the system does not require islanding, Fig. 7 compares the risks without and with islanding. It is shown that the risk of the system without islanding (dashed line) remains constant and with a value equal to zero as the fault does not cause any lost of load (despite no additional control actions are taken), i.e., the risk without islanding is equal to zero. Fig. 7 also highlights that the risk with islanding (solid line, calculated in parallel to the risk without islanding) remains constant, but in this case with a value of $18,900 \$ / h$.

It must be noticed that the risk with islanding in this case is introduced by the spurious operation of the scheme, as the result of the load shedding needed to recover the system after the islanding solution was implemented. Although the risk with islanding is relatively low $(18,900 \$ / h)$, Fig. 7 demonstrates that the proposed risk-based methodology for the when to island problem is effective as the risks do not cross (the risk with islanding is never lower than the risk without islanding).

\section{B. Case Study II: Islanding Required}

Case study II illustrates the methodology when islanding is actually required. A reduction of $9 \%$ of the total system load was made to increase the likelihood of instability following a disturbance. This case considers a severe fault cleared by backup protections that disconnects several transmission lines. As expected, such event weakens the system and is probable to lead to a blackout if no control actions are taken.

It is considered that at time $t=0.1 \mathrm{~s}$, a three phase-toground solid fault occurs near bus 23 at line $23-24$, and is cleared after remote back-up protections open the lines $22-23$, $23-25,23-32,24-70$ and $24-72$ at $t=0.5 s$. Note that these are all lines connected to buses 23 and 24 . According to the security criteria adopted in the work (used Costa Rica), remote back-up protections do have a delay and do not trip before $0.4 \mathrm{~s}$ giving sufficient time for local relays to operate. If no control action is undertaken, it can be observed in Fig. 8(a) that the system loses synchronism at approximately $0.4 s$ (two coherent groups of generators can clearly be seen). In Fig. 8(b) it can be noted that generators $G 10, G 12, G 25, G 26, G 31$ accelerate. In terms of the system voltages, Fig. 8(c) shows that the voltage magnitudes at the system buses are considerably low. Therefore, it can be concluded that the power system given the conditions analyzed in case study II requires to be split into islands to prevent a blackout.

The results of implementing the risk-based methodology are presented in Fig. 9. In terms of the risk of the system without islanding (dashed line), it is shown that this is low before the fault is cleared (i.e., when back-up protections open the lines mentioned above), highlighting that during this period is better to maintain the system connected, i.e., no islanding operation. However, Fig. 9 presents that approximately at $0.5 \mathrm{~s}$, this risk increases significantly to a value of $92,780 \$ / h$, that, in turn, indicates that a large-area blackout has occured in the system.

In parallel, the risk with islanding (given a solution found by the ICI methodology, see section II-C) is calculated. The risk with islanding shown as solid line in Fig. 9 highlights that a few milliseconds after the fault is cleared (i.e., at $t=0.51 \mathrm{~s}$ given that a fault cleared by back-up protections is very likely to lead to blackouts), the risk with islanding becomes smaller $(4,756 \$ / h)$ than the risk without islanding $(92,780 \$ / h)$. Thus, according to the risk-based methodology proposed in this work (see section III), this time is considered to be the most suitable moment to split the power system into two sustainable islands (given that two coherent groups of generators were created after the disturbance, see Fig. 8). 


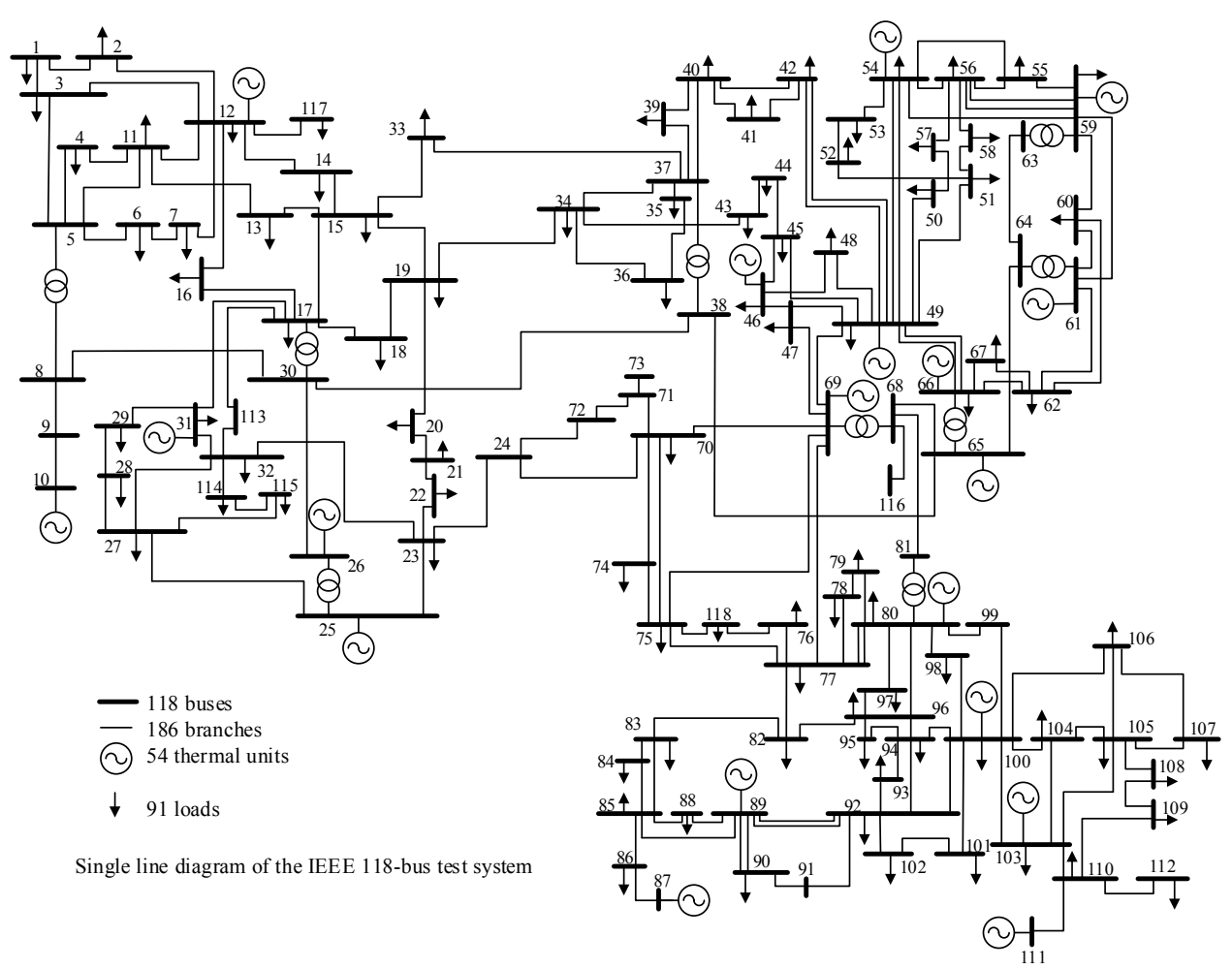

Figure 5. IEEE 118 bus test system
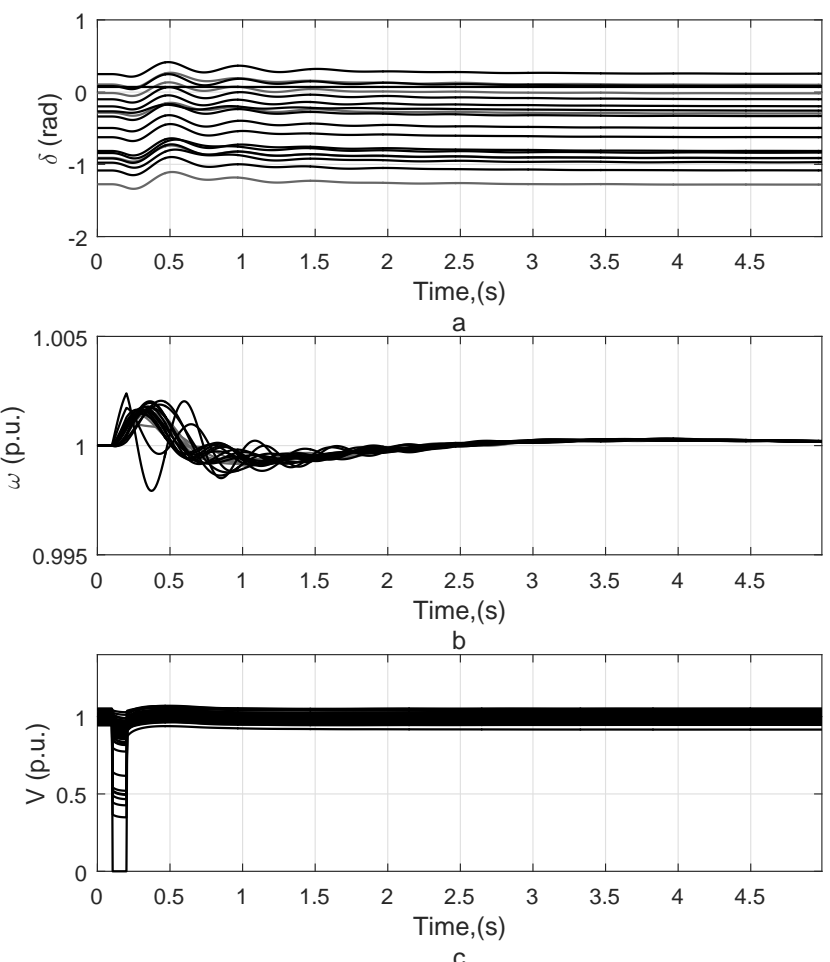

c

Figure 6. Case study I: Response of the system without ICI scheme

To demonstrate that the time for islanding determined by the risk-based methodology effectively prevents a blackout, the dynamic response of the system is now discussed. To cater for the delay in communications and the actual operation of the CBs, the actual islanding is implemented $40 \mathrm{~ms}$ after identifying that islanding is needed. Fig. 10 shows that the

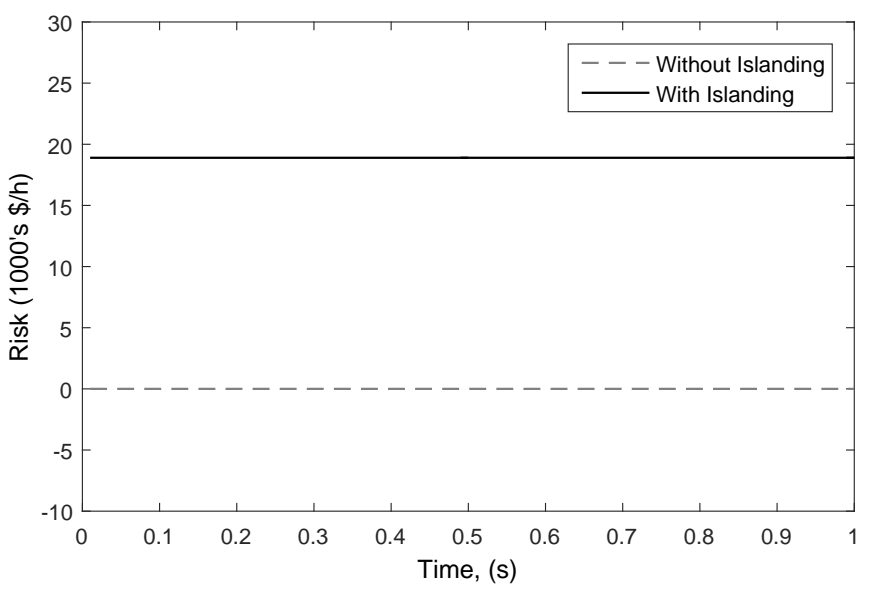

Figure 7. Case study I: Risk assessment

implementation of the islanding solution (found by the ICI algorithm as $15-33,19-34,30-38$ ) at $t=0.55$ s (i.e., $0.51 s+40 \mathrm{~ms}$ ) effectively avoided the blackout. Fig. 10(a) shows that two islands are created, one for each coherent group of generators. Here, it can be noted that two stable islands are created. In Fig. 10(b) it can be seen that a group of generators accelerate but after the system is split they start to recover. Fig. 10(c) highlights that the voltage magnitudes at the system buses recover after splitting and they establish close to $1 p . u$. Therefore, it can be concluded the timely separation of the system into islands successfully avoids the blackout.

\section{Effects of a Delayed Activation of the Islanding Scheme}

A delay in the actual islanding can be defined by operators to enable the implementation of other control actions (e.g., 

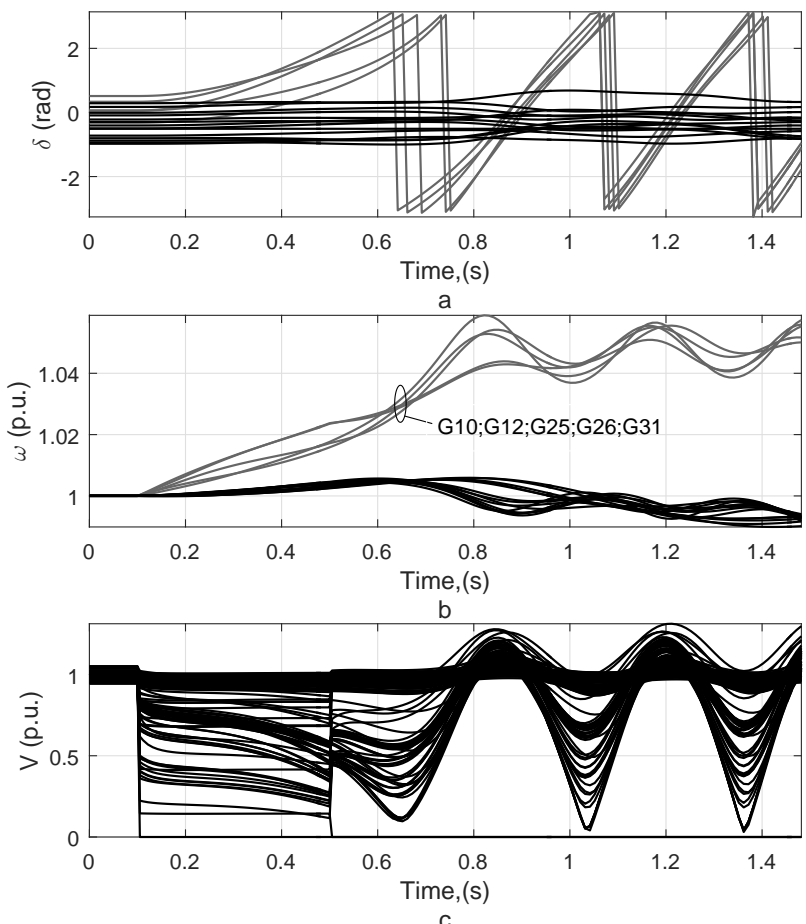

Figure 8. Case study II: Response of the system without ICI scheme

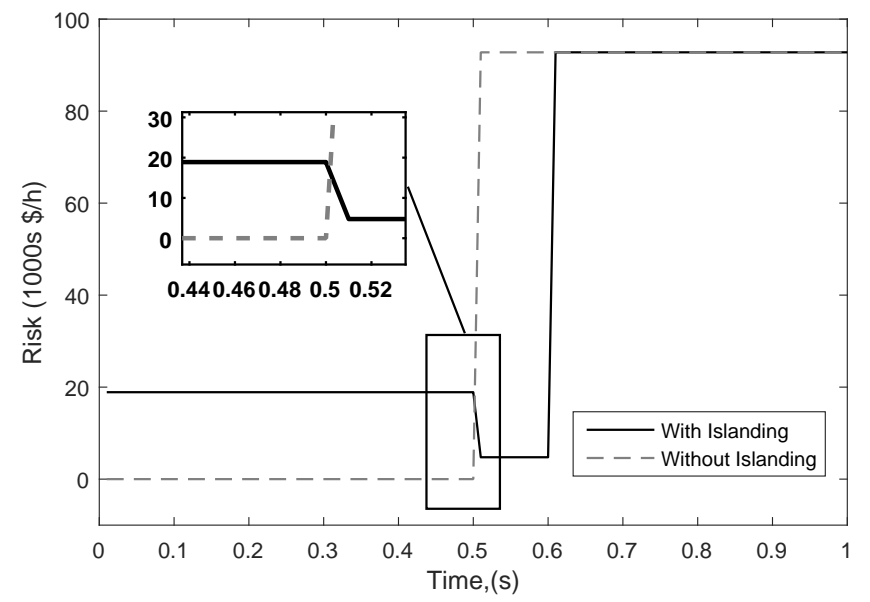

Figure 9. Case study II: Risk assessment

load shedding). Note that this delay is different from the one introduced by communications and the operation of $\mathrm{CBs}$ (defined above as $40 \mathrm{~ms}$ ). To understand the effects of an additional delay, this section uses Case Study II (see section IV-B) considering an operator-defined delay of $60 \mathrm{~ms}$. This means that the islanding signal that should have been sent at $t=0.51 \mathrm{~s}$ is actually triggered at $t=0.57 \mathrm{~s}$. Due to communications this is implemented at $t=0.61 \mathrm{~s}$. A delay of $60 \mathrm{~ms}$ has been arbitrarily selected and it is based on the risk assessment shown in Fig. 9.

Fig. 11 presents the dynamic response of the system when split at $t=0.61 \mathrm{~s}$ (i.e., $0.57 \mathrm{~s}+40 \mathrm{~ms}$ ). Fig. 11(a) shows that two islands are created (the first one with generators $G 10, G 12, G 25, G 26, G 31$ and the second one with the remaining ones), one for each coherent group of generators. The frequency displayed in Fig. 11(b) indicates that generators in the first island accelerate before islanding is implemented;
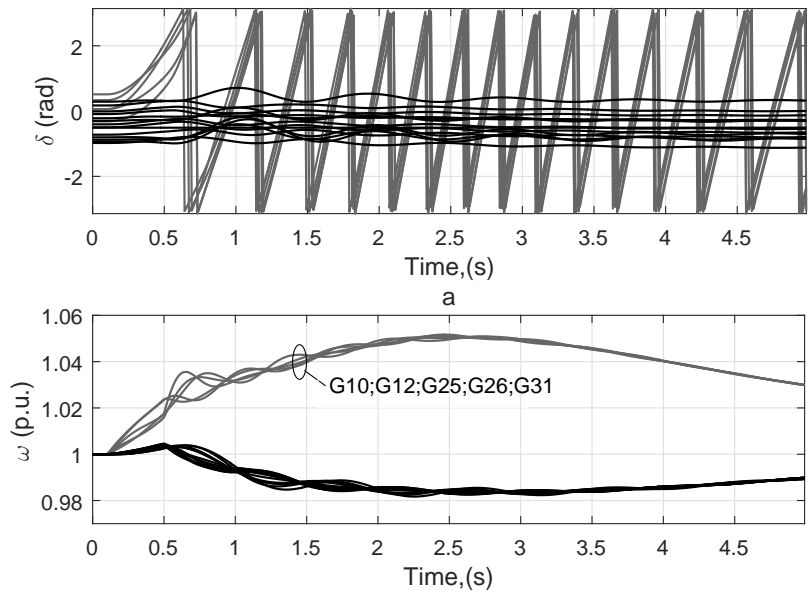

b

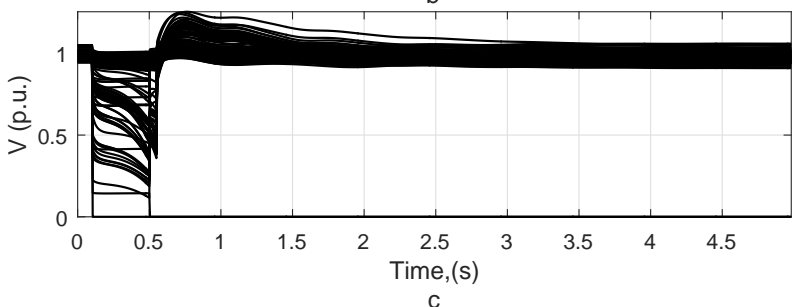

C

Figure 10. Case study II: Response of the system with ICI scheme at $t=$ $0.55 \mathrm{~s}$

in practice, this can lead to the trigger of overfrequency protection schemes. Crucially, Fig. 11(c) highlights that the voltage magnitudes at the system buses reach a significant low value, some of them near to $0.2 p . u$., which indeed indicates that a blackout has occurred in the system due to the relatively long delay introduced here $(60 \mathrm{~ms})$.

In conclusion, the investigation of a delayed implementation of islanding has demonstrated that if control actions are taken too late, the system might collapse. For the operation conditions presented in Case Study II, it can be observed in Fig. 9 that the longest delay that can be defined by operators is $50 \mathrm{~ms}$. This means that the actual islanding is implemented at $0.6 s=0.51 s+0.05 s+0.04 s$ (i.e., the most suitable time for islanding plus the delay introduced by operators plus the delay from communications and operation of $\mathrm{CBs}$ ).

\section{DISCUSSION}

The methodology proposed in this paper complements the well-studied where to island aspect, by addressing the question when to island through a risk-based analysis, thus resulting in an integral solution of the islanding problem. To quantify the risk in the obtained results, reliability data available in the literature was used. However, it is important to evaluate through a sensitivity analysis the potential effects of these parameters in the solution found by the methodology.

To estimate the impact, this work adopted the value of lost load as this is an acceptable and common metric. Nevertheless, other metrics, such as the social impact in final users as a result of this kind of severe events can be evaluated in future works.

As it was demonstrated in section IV, the speed of the where to island algorithm plays a critical role in a successful implementation of islanding. In this work a spectral clusteringbased algorithm [9] was selected for determining the islanding 

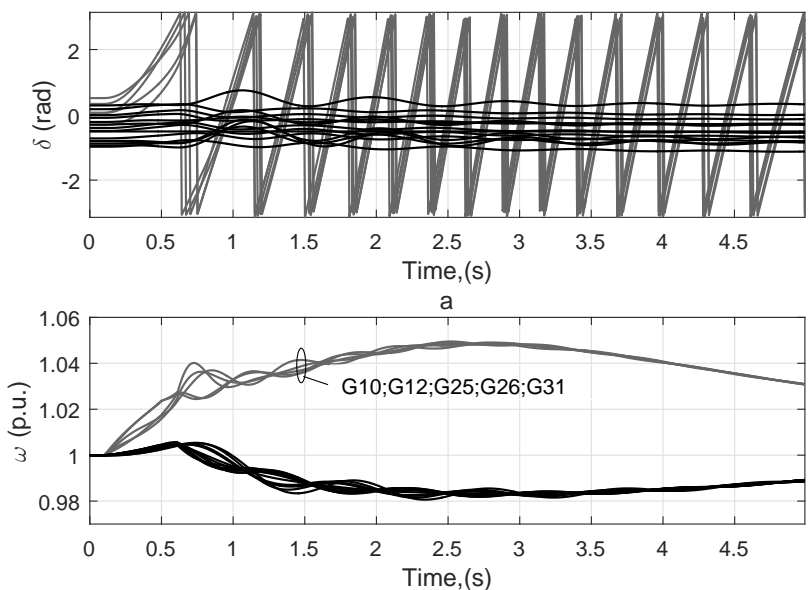

b

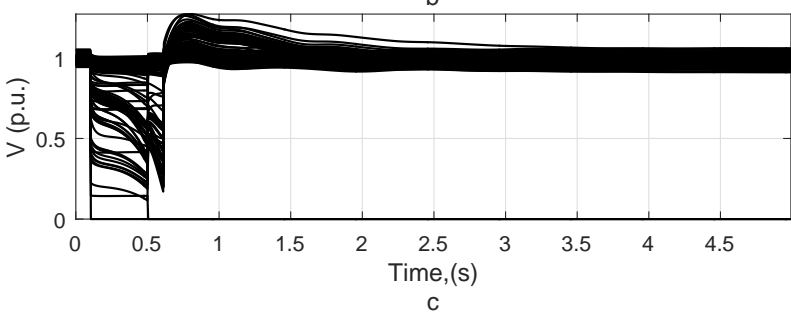

Figure 11. Response of the system with ICI scheme at $t=0.61 \mathrm{~s}$

solution. Nonetheless, any other algorithm for the definition of an islanding solution can be used, provided that this algorithm quickly determines an islanding solution.

Given that the investments are crucial for the implementation of new solutions, to truly implement the proposed methodology a cost-benefit analysis, considering the new acquisition or improving of infrastructure, communications, measurement devices (e.g., PMUs), could be performed.

\section{CONCLUSION}

This paper has proposed a risk-based methodology that compares the overall risk of the system without and with islanding (i.e., when an ICI scheme is in place) in order to define a suitable time for system splitting. Hence, this work addresses the when to island aspect in the intentional controlled islanding procedure, providing an integral solution to undertake islanding actions.

The methodology presented in this work is a novel approach, that considers the probability of the event, the reliability of both the grid components and information and communication technologies, as well as the potential economic costs to find a suitable solution. It is also a flexible method, that can be adapted to multiple contexts without affecting the outline of the procedure, e.g., different TSO requirements based on actual conditions of the system.

The proposed methodology has been tested using the dynamic model of the IEEE 118-bus system and the security criteria used by the system operator in Costa Rica. Timedomain simulations have been performed and it has been observed that the most suitable time for the creation of islands corresponds to the crossing point between the risks of the system without and with islanding, i.e., when the risk without islanding becomes larger than the risk with islanding.
Simulation results have shown that a timely implementation of islanding can reduce the system' risk, which, in turn, result in lower economic losses (from $92,780 \$ / h$ to $4,756 \$ / h$ in the analyzed test system), deeming the proposed methodology a very good tool for the decision making process of transmission system operators. Moreover, it was demonstrated that the methodology is able to find a solution in a frame of milliseconds (10 ms in this work), fast enough to avoid the blackout. Finally, it was exhibited that the more delay is introduce when creating the islands, the more likely the system is to the blackout due to lack of control actions.

\section{REFERENCES}

[1] L. Dulau, M. Abrudean, and D. Bica, "Effects of distribuited generation on electric power systems," in 7th International Conference Interdisciplinarity in Engineering (INTER-ENG 2013), 2014.

[2] M. Vaiman, K. Bell, Y. Chen, B. Chowdhury, I. Dobson, P. Hines, M. Papic, S. Miller, and P. Zhang, "Risk assessment of cascading outages: Methodologies and challenges," IEEE Trans. on Power Syst., vol. 27, no. 2, pp. 631-641, May 2012.

[3] W. Lu, Y. Bésanger, E. Zamai, and D. Radu, "Blackouts: Description, analysis and classification," in WSEAS International Conference on Power Systems, Sep 2006, pp. 429-434.

[4] K. Sun, K. Hur, and P. Zhang, "A new unified scheme for controlled power system separation using synchonized phasor measurements," IEEE Trans. on Power Syst., vol. 26, no. 3, pp. 1544-1554, Jan 2011.

[5] N. Senroy, G. Heydt, and V. Vittal, "Decision tree assisted controlled islanding," IEEE Trans. on Power Syst., vol. 21, no. 4, pp. 1790-1797, Nov 2006.

[6] N. Senroy and G. Heydt, "Timing of a controlled islanding strategy," Transmission and Distribution Conference and Exhibition, IEEE PES, pp. 1460-1466, 2006.

[7] M. Adibi, R. Kafka, S. Maram, and L. Mili, "On power system controlled separation,” IEEE Trans. on Power Syst., vol. 21, no. 4, pp. 1894-1902, Nov 2006.

[8] H. You, V. Vittal, and Z. Yang, "Self-healing in power systems: an approach using islanding and rate of frequency decline-based load shedding," IEEE Trans. on Power Syst., vol. 18, no. 1, pp. 174-181, Feb 2013

[9] J. Quirós-Tortós, R. Sánchez-García, J. Brodzki, and J. Bialek, "Constrained spectral clustering-based methodology for intentional controlled islanding of large-scale power systems," IET Generation, Transmission \& Distribution, vol. 9, no. 1, pp. 1-12, 2015.

[10] J. McCalley and W. Fu, "Reliability of special protection schemes," IEEE Trans. on Power Syst., vol. 14, no. 4, pp. 1400-1406, Nov 1999.

[11] M. Panteli, P. Crossley, and J. Fitch, "Quantifying the reliability level of system integrity protection schemes," IET Generation, Transmission \& Distribution, vol. 8, no. 4, pp. 753-764, Apr 2014.

[12] P. Fernández-Porras, M. Panteli, and J. Quirós-Tortós, "A risk-based methodology for defining the time of intentional controlled islanding," in Innovative Smart Grid Technologies Latin America (ISGT LATAM), 2015 IEEE PES, Oct 2015, pp. 828-832.

[13] Regulatory Authority of Public Services (ARESEP), "Planning, operation and access to the national electric system (ar-nt-poasen-2014, in spanish)," Costa Rica, Tech. Rep., 2014.

[14] W. Li and J. Q. Zhou, "Probabilistic reliability assessment of power system operations," Electric Power Components and Systems, vol. 36, pp. 1102-1114, Sep 2008.

[15] W. Vesely, F. Goldberg, N. Roberts, and D. Haas1, Fault Tree Handbook, N. R. Commission, Ed. NUREG-0492, 1981.

[16] U. Von Luxburg, "A tutorial on spectral clustering," Statistics and Computing, vol. 17, no. 4, pp. 395-416, Nov 2007.

[17] T. Hsiao and C. Lu, "Risk informed design refinement of a power system protection scheme," IEEE Trans. on Reliability, vol. 57, no. 2, pp. 311321, Jun 2008.

[18] P. Demetriou, M. Asprou, J. Quiros-Tortos, and E. Kyriakides, "Dynamic ieee test systems for transient analysis," IEEE Systems Journal, vol. PP, no. 99, pp. 1-10, Jul 2015.

[19] DIgSILENT PowerFactory, ed. Heinrich-Hertz-Straße, Germany.

[20] MATLAB R2010a: Natick, Massachusetts: The MathWorks Inc., 2010. 